\title{
Towards genome-based and environment-informed breeding intensification
}

\author{
Samsonova M. \\ Peter the Great St. Petersburg Polytechnic University, St. Petersburg, Russia \\ e-mail:m.samsonova@spbstu.ru
}

Chickpea is the second most widely grown food legume, providing a vital source of nutritional nitrogen for $\sim 15 \%$ of the world's population. With success in chickpea domestication came an incremental loss in adaptive variation and currently genetic and adaptive variation has nearly disappeared from elite high-yielding chickpea cultivars. Given this perspective there is a compelling need to re-introduce genetic variation into chickpea lines. The Vavilov seed bank contains numerous landraces collected nearly hundred years ago, before intensive breeding of most crops, and thus is a potential reservoir of "genomic gems" that might offer solutions for improving elite varieties. Here we analyze the genomes and ancestral environments of $\sim 500$ of Vavilov's original landraces, sampled from major historic centers of chickpea cultivation and secondary diversification. We dissect important phenotypes to their underlying genetic basis, including large-effect genes and whole-genome smaller-effect contributions, enumerate beneficial alleles, identify polymorphisms that assort by environment and that are candidates for local adaptation and figure how to this standing variation can be combined to produce improved cultivars. This knowledge could then be used to optimize chickpea breeding. 